\title{
Investigation of HP- $\beta-C D$ Inclusion Compound Preparation Process for Pulsatilla Chinensis (Bunge) Regel Total Saponins1
}

\author{
Zhenhua Chen ${ }^{1, *}$, Suzhen Liu ${ }^{1}$, Yongmei Guan ${ }^{2}$, Ni Zhang ${ }^{2}$, Ming Yang ${ }^{2}$ \& \\ Hongning Liu $^{2}$ \\ ${ }^{1}$ School of Pharmacy, Jiangxi Science and Technology Normal University, Nanchang, 330013,
China \\ ${ }^{2}$ Key Laboratory for Modern preparation of TCM, Ministry of Education; Jiangxi University of \\ Traditional Chinese Medicine, Nanchang, 330004, China
}

zhenhuadeai@163.com

Keywords: Pulsatilla chinensis (Bunge) Regel total saponins, HP- $\beta-C D$, inclusion compound.

\begin{abstract}
This paper used parallel test of single factor to investigate feed ratio of inclusion compound, drying method, the volatilization temperature of ethanol for Pulsatilla Chinensis (Bunge) Regel total saponins-HP- $\beta$-CD inclusion compound. The results showed that the best preparation process of inclusion compound was as following: the feed ratio of Pulsatilla total saponin extract and HP- $\beta$-CD was 1:1; After the Pulsatilla total saponins extract with ethanol solution was dropwise added to the HP- $\beta$-CD aqueous solution, the mixed solution was continuously stirred for $6 \mathrm{~h}$ at $60 \mathrm{C}$ and was freeze-dried for $12 \mathrm{~h}$. The preparation process for Pulsatilla Chinensis (Bunge) Regel total saponins-HP- $\beta$-CD inclusion compound was simple and it is helpful to improve the drug dissolution.
\end{abstract}

\section{Introduction}

Pulsatilla chinensis (Bunge) Regel can clear away heat and detoxicate, and can cool blood and arrest dysentery, which activities especially in cleaning colon heat and blood toxic heat. Pharmacological studies confirmed that Pulsatilla chinensis (Bunge) Regel extract had a good effect on anti-ulcer colitis [1], which can be developed as an oral colon-targeted preparation.

Pulsatilla chinensis (Bunge) Regel total saponins was extracted by ethanol from Pulsatilla chinensis (Bunge) Regel and separated and purified by Macroporous resin. The preliminary results showed that it had a low solubility in water [2] and was difficult to release when it was made into pellets directly. However, when it was made into hydroxypropyl- $\beta$-cyclodextrin (HP- $\beta$-CD) inclusion compound, it can significantly enhance the dissolution rate of the drug [3]. This paper had examined the prescription and process of Pulsatilla chinensis (Bunge) Regel total saponins-hydroxypropyl- $\beta$-cyclodextrin inclusion compound, which can lay the foundation on preparing pellets further.

\section{Methods and results}

\subsection{Instruments and materials}

The instruments used together with the suppliers were as follows: Freeze Dryer (FDU-1100, TOKYO RIKAKIKAI Co. Japan); Vacuum oven (DZF-6050, Medical Devices Ltd. Emerging Artists, Shanghai); HPLC (Agilent 1200, Agilent Technologies Ltd. USA).

The materials used together with the suppliers were as follows: Pulsatilla chinensis (Bunge) Regel total saponins (National Engineering Research Center for Manufacturing Solid Preparation of Traditional Chinese Medicine, Lot:20110804); Pulsatilla saponin D reference (National Engineering Research Center for Manufacturing Solid Preparation of Traditional Chinese Medicine, Lot: 
20111108); Hydroxypropyl- $\beta$-cyclodextrin (HP- $\beta$-CD, Biologica Chemical Ltd. Xian, Deli); Chromatography acetonitrile (Sinopharm Chemical Reagent Ltd.); Ethanol (Sinopharm Chemical Reagent Ltd.); Distilled water (Laboratory homemade). All other chemicals were of analytical-reagent and deionized double-distilled water was used throughout the study.

\subsection{Establishment of the HPLC method}

Hypersil ODS2 C18 column $(5 \mu \mathrm{m}, 250 \mathrm{~mm} \times 4.6 \mathrm{~mm}$, Dalian Elite Ltd.), mobile phase of acetonitrile- $0.1 \%$ phosphoric acid solution (39:61), flow rate $1 \mathrm{~mL} \cdot \mathrm{min}-1$, column temperature 30C, detection wavelength $203 \mathrm{~nm}$, injection volume $0.02 \mathrm{~mL}$.

Number of theoretical plates calculated by the peak of Pulsatilla saponin D was not less than 5000. Index components from Pulsatilla saponin D had no interference on the peak position and had better separation and peak shape, and its chromatography was shown in Figure 1.

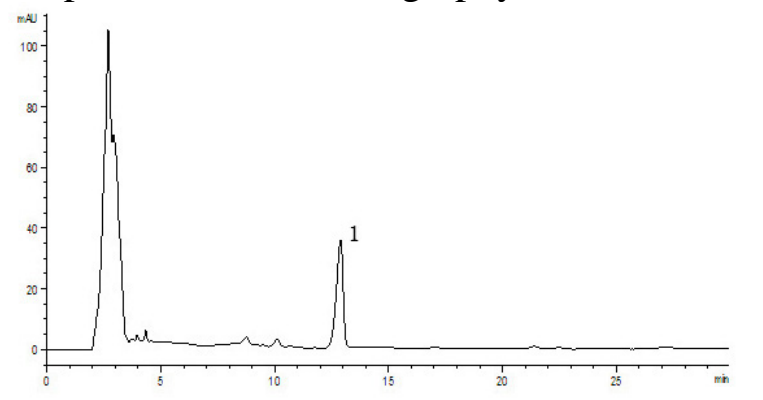

A
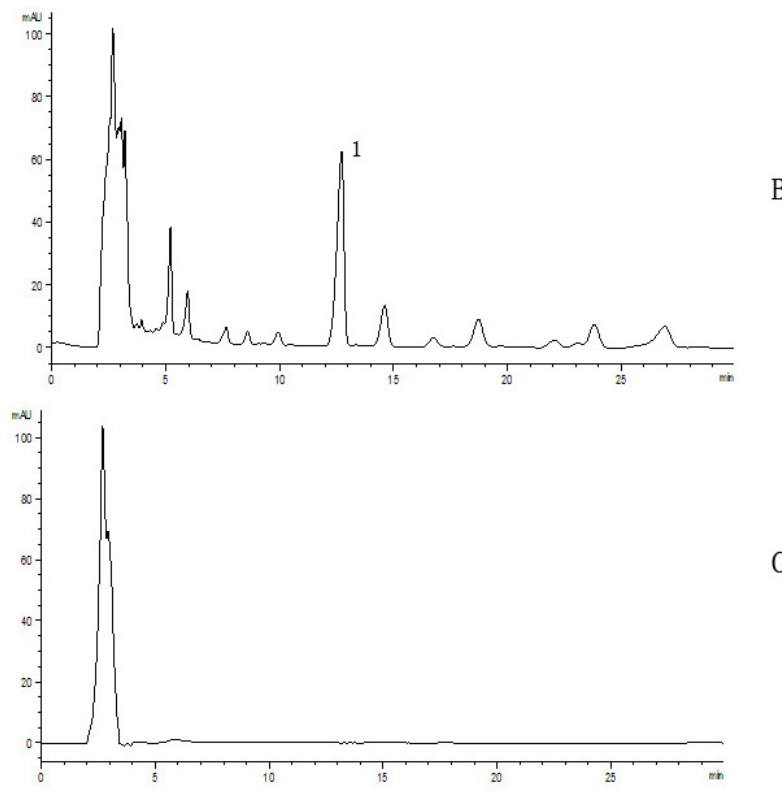

A: reference; B: test; C: blank solvent;

1: Pulsatilla saponin D

Fig.1: HPLC chromatogram

About 20mg Pulsatilla saponin D reference was accurately weighed, and was dissolved in methanol to $10 \mathrm{~mL}$ as a stock solution. A volume of $0.25,0.5,1.0,1.5,2.5 \mathrm{~mL}$ of the stock solution was accurately absorbed and then was respectively added methanol to $5 \mathrm{~mL}$. The prepared solution was injected according to the chromatographic conditions mentioned above and the results were recorded. The linear regression equation was obtained by taking concentration $C(g \cdot L-1)$ as the vertical axis and the peak area $\mathrm{A}(\mathrm{mAU} \cdot \mathrm{min})$ as the abscissa, and the results were shown as following: $A=4902.6 C+8.926$, the RSD was $r=0.9995$ and the linear range was $0.1009 \sim 1.009$ $\mathrm{g} \cdot \mathrm{L}-1$.

$1 \mathrm{~mL}$ sample solution, of which the content had been determined, was accurately measured, and about 10mg reference was accurately added into it. Then the methanol was added to the volume to $25 \mathrm{ml}$. The same method was used to prepare three parallel solutions. The prepared solution was 
determined according to the chromatographic conditions above. The results showed that the calculated recovery was $97.45 \%$ and the RSD was $1.43 \%$.

The same sample solution was measured for three times on the same day and was continuously measured for three days according to the chromatographic conditions above. The intra-day and inter-day precision were respectively calculated, and the results were $0.13 \%$ and $0.52 \%$, respectively.

\subsection{Investigation of feeding ratio}

About 3.0g Pulsatilla chinensis (Bunge) Regel total saponins was weighed, and the weighed sample was placed in a conical flask. $60 \mathrm{~mL}$ ethanol was added into the conical flask. And then the sample was dissolved by ultrasound and heating. The HP- $\beta-C D$ (2.0, 3.0 and 4.5g) was weighed, and was respectively placed in the $100 \mathrm{~mL}$ beaker. Appropriate amount of distilled water was added into each beaker. The sample of each beaker was dissolved by magnetic stirring. The Pulsatilla chinensis (Bunge) Regel total saponins ethanol solution was dropwise added when the HP- $\beta$-CD solution was heated to 60C. After all the solution was added, the temperature was maintained at $60 \mathrm{C}$ for about $2 \mathrm{~h}$. Then the mixed solution was cooled to room temperature and was continually stirred until no alcohol taste. The final mixed solution was dried and crushed to obtain the Pulsatilla total saponins-HP- $\beta$-CD inclusion compound.

According to the third method of dissolution assay method in Chinese Pharmacopoeia (2010) bipartite appendix XC, the dissolution of the inclusion compound was respectively determined. Determination of the index components in the dissolution sample filtrate was according to the HPLC analysis method mentioned above. The results of the calculated dissolution were shown in Figure 2.

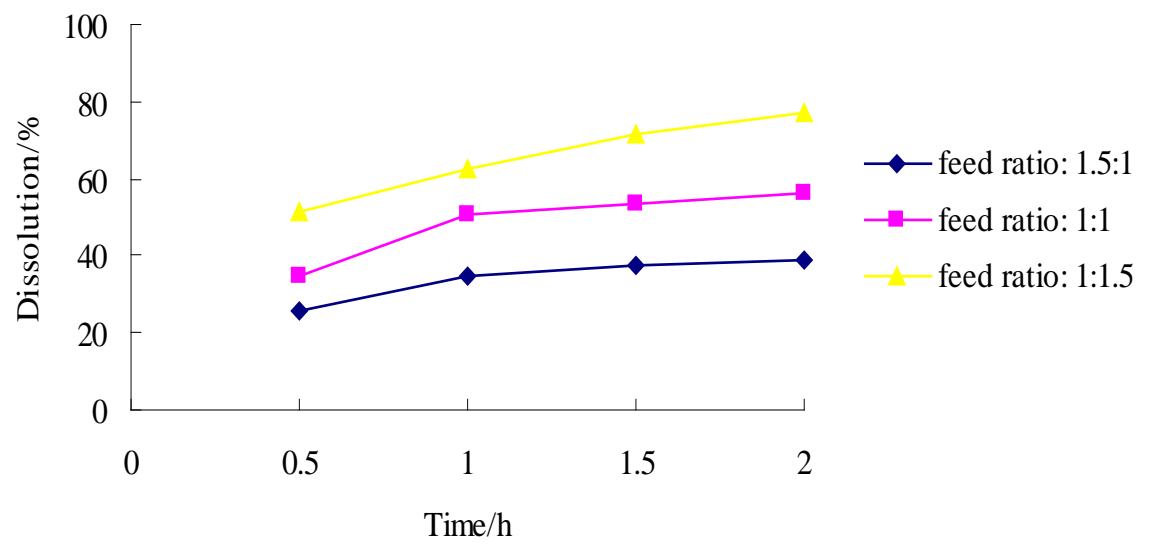

Fig 2. The dissolution curve of cyclodextrin inclusion compound of different feeding ratio The results showed that after the hydroxypropyl- $\beta$-cyclodextrin inclusion compound was prepared, the drug dissolution was accelerated significantly in the tested range. The higher proportion of hydroxypropyl- $\beta$-cyclodextrin, the more significantly the drug dissolution accelerated. Considering the influence of the drug content and solubilization and other factors, we can ultimately determine that the best feed ratio was Pulsatilla chinensis (Bunge) Regel total saponins and HP- $\beta$-CD of 1:1 for the preparation of inclusion compound.

\subsection{Selection of drying methods}

The inclusion compound was prepared by solution stirring method with the ratio of Pulsatilla chinensis (Bunge) Regel total saponins and HP- $\beta$-CD quality of 1:1. The freeze drying method and vacuum drying method were respectively used for the preparation of the inclusion compound, and the drying time was recorded. The characteristics of dried product were observed and the dissolution was determined. The investigative results were shown in Table 1 . The results showed 
that the prepared cyclodextrin inclusion compound from the two drying methods had no significant differences in the solubilization, but the required time of freeze-drying method was shorter and the dried product was looser, which had benefit for the preparation molding.

Table 1: Study results of different drying methods

\begin{tabular}{cccc}
\hline Drying Method & Drying Time & $\begin{array}{c}\text { Product } \\
\text { Characters }\end{array}$ & Dissolution at 1h \\
\hline $\begin{array}{c}\text { Freeze-drying } \\
\text { method }\end{array}$ & $12 \mathrm{~h}$ & loose & $71.3 \%$ \\
$\begin{array}{c}\text { Vacuum drying } \\
\text { method }\end{array}$ & 48h above & dense & $70.4 \%$ \\
\hline
\end{tabular}

\subsection{Selection of ethanol evaporation temperature}

The cyclodextrin inclusion compound was prepared by the method described above with the quality ratio of Pulsatilla chinensis (Bunge) Regel total saponins and HP- $\beta-C D$ of 1:1. The prepared cyclodextrin inclusion compound was respectively stirred at 50, 60, 70C until no alcohol taste and then dried by freeze-drying. The required time of ethanol evaporation was recorded and the dissolution was determined. The results were shown in Table 2. The dissolution of the prepared cyclodextrin inclusion compound was accelerated with the reduction of the temperature, but the preparation time of the cyclodextrin inclusion compound was extended accordingly. The cyclodextrin inclusion compound was prepared at 60C and 50C had little differences in dissolution and both were significantly better than that at 70C, but the preparation time at 60C was significantly shorter than that at 50C. Considering the preparation time and solubilization effect, the volatile ethanol of 60Cwas selected for the preparation process of inclusion compound.

Table 2: Investigation of ethanol evaporation temperature

\begin{tabular}{ccc} 
Temperature / C & Preparation Time / h & Dissolution at 1h \\
\hline 50 & 12 & 77.2 \\
60 & 6 & 72.1 \\
70 & 4 & 60.2 \\
\hline
\end{tabular}

\section{Conclusion}

The prepared inclusion compound by the process in this paper can significantly improve the dissolution of the drug. Meanwhile, the low temperature for the evaporation of ethanol was good for the increase of the dissolution rate of inclusion compound, which suggested that the inclusion effect for Pulsatilla chinensis (Bunge) Regel total saponins and HP- $\beta-C D$ was reflected to be exothermic reaction.

\section{Acknowledgment}

This work was supported by a grant from the Natural Science Foundation of Jiangxi Province (20122BAB215041), the Doctoral Scientific Research Foundation of Jiangxi Science and Technology Normal University (3000990117), the Key Laboratory of Drug Design and Optimization of Jiangxi Science and Technology Normal University (300098010306), and partially by National Scientific and Technology Major Special Project for Significant New Drug Creation (2013ZX09103002-001). 


\section{References}

[1] Wenyuan Zhang, Shengxi Han, Hong Yang, Studies on anti-inflammatory mechanism of Pulsatilla alcohol extract on dextran sulfate sodium induced colitis, Chinese Journal of Digestion. 24 (9), pp.568-570, 2004.

[2] Zhenhua Chen, Yongmei Guan, Ni zhang, etc., Investigation of dissolvability of total saponins from Pulsatilla Chinesis, Chinese Journal of Experimental Traditional Medical Formulae. 18 (13), pp.28-30, 2012.

[3] Yongmei Guan, Ni zhang, Zhenhua Chen, etc., Study on solubilization effect of solid dispersion technology and inclusion technology on Pulsatillae total saponins, China Journal of Chinese Materia Medica. 38 (23), pp.4061-4066, 2013. 